\title{
Groundwater modeling for the Mekong Delta using iMOD
}

\author{
Peter Vermeulen $^{a}$, Nguyen Hong Quan ${ }^{\text {b }}$, Nguyen Dinh Giang Nam ${ }^{\text {c, }}$ \\ Pham Van Hung ${ }^{d}$, Nguyen Tien Tung ${ }^{d}$, Tong Viet Thanh ${ }^{\mathrm{e}}$, Rien Dam ${ }^{a}$ \\ ${ }^{a}$ Department of Groundwater Management, Deltares, the Netherlands \\ ${ }^{b}$ Institute for Environment and Resources (IER) - Vietnam National University, Ho Chi Minh City \\ ${ }^{c}$ Department for Environment and Natural Resources Management, Can Tho University, Vietnam \\ ${ }^{d}$ Division for Water Resources Planning and Investigation for the South of Vietnam (DWRPIS) \\ ${ }^{e}$ Division of Water Resources and Minerals, Dep. of Natural Res. and Environment of Ho Chi Minh City \\ Email: peter.vermeulen@deltares.nl
}

\begin{abstract}
Groundwater (together with surface water) is essential to human live and socio-economic development in the Mekong Delta, Vietnam. It is extensively used by local people in the last decades especially for drinking water, agriculture and industry. Knowledge on the impacts on water systems by natural (e.g. climate change, sea level rise, and/or discharge variations from the Mekong River) as well as anthropogenic processes (e.g. increased population, agriculture, industry, land cover changes, and man-induced land subsidence) is still limited. In addition, (ground)water availability and variability as a function of time, is unclear. For these purposes, a supra-regional 3D groundwater flow model for the whole Mekong Delta using iMOD was developed. iMOD stands for Interactive MODeling and facilitates an easy-to-use modeling environment to engage stakeholders and stimulate participation in active groundwater management. Another difference compared to conventional modeling tools, is the generic geo-referenced data structure that may contain files with unequal resolutions and that can be used to generate sub-models at different scales and resolutions applying up- and downscaling concepts. This is done internally without creating sub-sets of the original model data. For modelers and stakeholders, this offers high performance, flexibility and transparency. We describe and demonstrate the flexibility and up- and downscaling abilities of iMOD by means of two local refinements for Ho Chi Minh City and Can Tho City.

Suppose the modeler needs to simulate groundwater flow for the total area covered by the data set, but the theoretical size of the model is far too big to fit in any CPU-memory. iMOD facilitates generating sub models for parts of the whole area of interest with a user-defined resolution depending on how large the available CPU-memory is and how long the modeler permits her/himself to wait for the model calculations to last. To generate a high resolution result for the whole model domain, a number of partly overlapping but adjacent sub models are invoked and the result of the non-overlapping parts of the models are assembled to generate the whole picture. The modeler should of course be cautious that the overlap is large enough to avoid edge effects, but this overlap is easily adjustable in iMOD. A big advantage of this approach is that running a number of small models instead of running one large model (if it would fit in memory, which it often will not) takes much less computation time; computation time $(\mathrm{T})$ depends on the number of model cells (n) exponentially: $\mathrm{T}=\mathrm{f}\left(\mathrm{n}^{1,5-2,0}\right)$. iMOD also allows the utilization of parallel computing, but this is not obligatory. This approach allows the modeler to interactively generate models of any sub-domain within the area covered by the data set. Using this approach means that the modeling workflow is very flexible and not limited anymore by hardware when utilizing iMOD. This enables the IER, Can Tho University, DWRPIS and DWRM to proceed with building and improving (local) groundwater models in the Mekong Delta in any order, region after region or sub regions within larger regions with resolutions that fit the actual needs and still maintain consistency between the different sub models and resolutions.
\end{abstract}

Keywords: $\quad$ iMOD, Mekong Delta, Vietnam, Groundwater Flow Modeling 


\section{INTRODUCTION}

The Mekong Delta is one of the most vulnerable regions in the world for the impacts of climate change and sea level rise (Syvitski, Kettner et al., 2009; IPPC, 2012). Given an intensive surface water network, water is allocated for different activities in the region like households, industry, agri- and aquaculture, and transportation. Nevertheless, due to the high variation (in quantity and quality) of surface water distribution in time and space, the use of other sources including rain water and groundwater is common in the region. Groundwater as source in the Mekong Delta is extensively used in the last decades (Eugene, 1971; IUCN, 2011). A compiled paper by IUCN (2011) showed that groundwater in the Mekong Delta supplies water for domestic use, urban water supply, irrigation, aquaculture, and industrial sites. For example, 24 per cent of the population of Can Tho City, the largest city in the Delta, uses groundwater for domestic use. Moreover, during the dry season groundwater is also used for irrigation purposes. It is estimated, but highly variable and uncertain, that there are 465,000 groundwater wells and a total extraction of 1,229,000 $\mathrm{m}^{3} /$ day. Figure 1 shows the drawdown in several wells in Can Tho City $(1993-2011)$ and in the $\mathrm{Ca} \mathrm{Mau}$ peninsula (1995 2004) that decrease $0.3-0.5 \mathrm{~m}$ per year. Over extraction causing groundwater drawdown is one of the clear phenomena in the area. In addition, saltwater intrusion, and pollution are problems related to groundwater use (Danh 2008; Wagner, Bui et al. 2012). Moreover, land subsid-

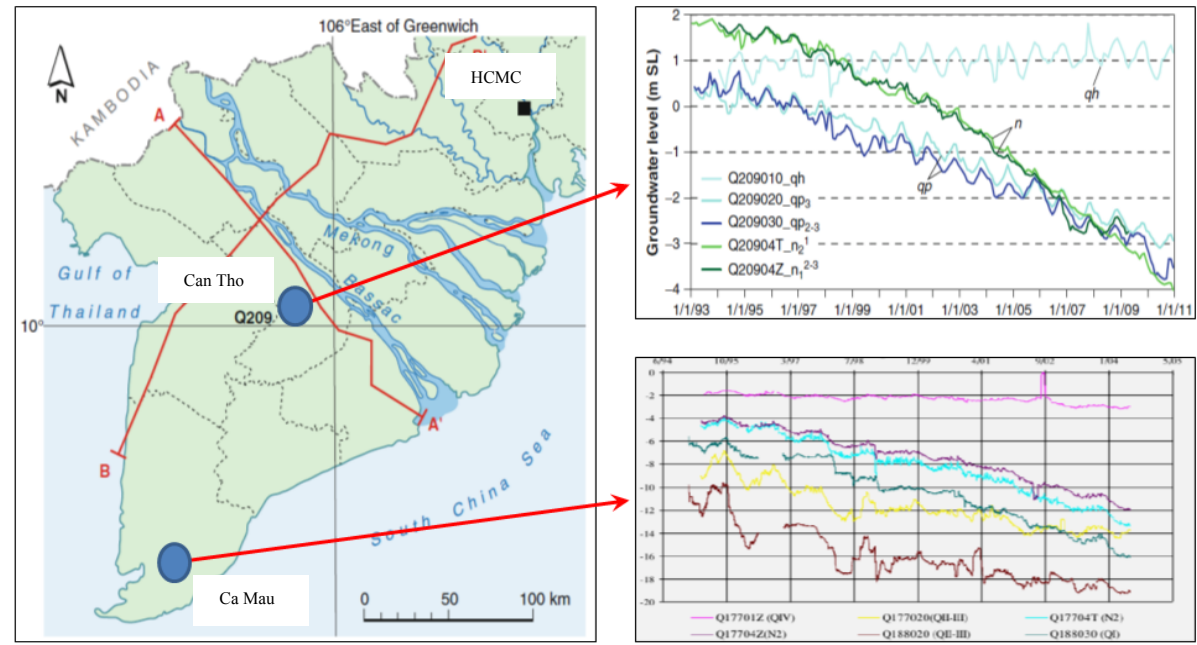

Figure 1. Time series of groundwater level fluctuation in Mekong Delta, modified from (Wagener et al, 2012) and (IUCN, 2011).

ence and sea-level-rise may have potential negative impacts on the groundwater resource situation in the Mekong Delta. Therefore, developing a versatile tool for improved large-scale groundwater management in the Delta would be very helpful.

A mathematical model is very essential in water resources management in general and in groundwater management in particular (Anderson and Woessner, 1992). The appropriate model settings will help in developing suitable management scenarios to prevent groundwater depletion, which is the key for sustainable groundwater use. However, model applications in the Mekong Delta that investigate groundwater variability as well as how natural and anthropogenic factors influence this system are still rather limited. The first attempt was done by Haskoning B.V., DWRPIS (Boehmer, 2000) to set up a regional groundwater model for the Nam Bo region including the Vietnamese Mekong Delta. The model was used to study the groundwater flow in the aquifers, to determine the recharge mechanism, as well as to determine the groundwater potential for drinking water supply. Since the model setup, a number of activities were done to update the model, e.g. inclusion of a renewed regional hydrological map (DGMS, 2004) and the increased number of groundwater extraction wells during the last decade. It is expected that these kinds of changes and improvements are occurring more and more in the future and therefore it urges for a modeling environment that easily adapts any improvements and changing user needs, and is transparent in its data requirements and usage. In this paper, we describe the philosophy of modeling with iMOD which is completely different from methodologies that are used in other conventional software packages. iMOD is applied to fill in a database (individual files) with relevant information on the finest scale available to setup a groundwater flow model for the Mekong Delta. The model purpose is to study the potential groundwater distribution in time and space of the Mekong Delta. Thereafter, we zoom in for two different areas (Ho Chi Minh City and Can Tho City) and add more detailed information to be able to compute the drawdown in more detail (Can Tho City) and/or the interaction between groundwater and surface water (Ho Chi Minh City). In the final part, the paper presents our perspectives for improving the model as well as what can be further applied to cope with current and future challenges. 


\section{LEAVING THE ERA OF BUILDING SERIES OF INDIVIDUAL MODELS BEHIND}

High resolution groundwater flow modeling, necessary to evaluate effects on a local scale, has traditionally been restricted to small regions given the computational limitations of the CPU memory to handle large numerical MODFLOW-grids. Although CPU-memory size doubles every two years ('Moore's law') the restriction still holds from a hardware point of view. This restriction has traditionally forced a model builder to always choose between (1) building a model for a large area with a coarse grid resolution or (2) building a model for a small area with a fine grid resolution. For some time it appeared that finite element models could fill the gap by refining the grid only where hydrological gradients were anticipated. However, unanticipated stress may also occur in parts of the model area where the grid is not yet refined resulting in a possible undesired underestimation of these effects. Theoretically, the modeler could choose to design a finite element network with a high resolution everywhere, but then it becomes more economical to use finite differences. This is why Deltares has based its innovative modeling techniques on MODFLOW considering it is largely seen world-wide as the standard finite difference source code. Still, modelers ideally need an approach that allows: (1) flexibility to generate high resolution model grids everywhere when needed, (2) flexibility to use or start with a coarser model grid, (3) reasonable runtimes / high performance computing and (4) conceptual consistency over time for any part of the area within their administrative boundary. Deltares has invested in understanding all of these requirements and has developed the iMOD software package to advance the methods and approach used by modelers and regulators.

\section{THE IMOD APPROACH}

The development of the iMOD approach took off in The Netherlands in 2005 when Deltares and a group of 17 stakeholders decided to jointly build a numerical groundwater model for their common area of interest (Berendrecht, Snepvangers et al., 2007; Vermeulen, 2013). The groundwater model encompasses the entire north of the Netherlands at a resolution of $25 \mathrm{~m}$ and was constructed together via an internet accessible userinterface. This makes it possible for the modelers to easily access the model data, intermediate results and participate in the model construction. The iMOD approach allows gathering the available input data to be stored at its finest available resolution; these data don't have to be clipped to any pre-defined area of interest or pre-processed to any model grid resolution (Figure 2).

Resolutions of parameters can differ and the distribution of the resolution of one parameter can also be heterogeneous. In addition, the spatial extents of the input parameters don't have to be the same. iMOD will perform up- and down scaling (Vermeulen, 2006) whenever the resolution of the simulation is lower or higher than that of the available data. This approach allows the modeler to interactively generate models of any subdomain within the area covered by the data set. When priorities change in time (e.g. due to changing political agenda's) the modeler can simply move to that new area of interest and apply any desired grid resolution. In addition the modeler can edit the existing data set and / or add new data

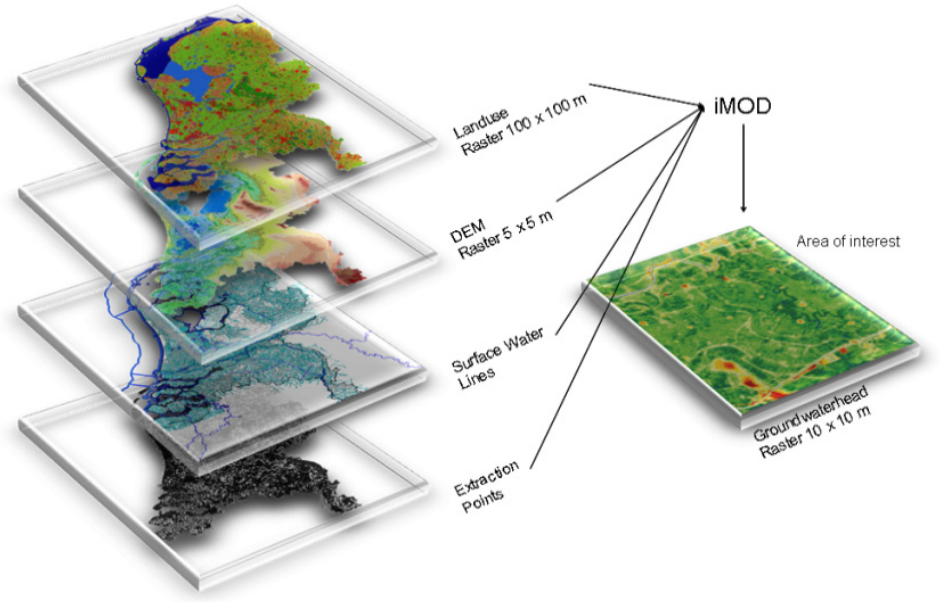

Figure 2. The iMOD-approach: one input data set without clipping to any areas of interest.

types to the data set. Utilizing the internal up- and down-scaling techniques ensures that sub-domain models remain consistent with the bigger regional model or that the regional model can locally be updated with the details added in the sub-domain model (Minnema, Vermeulen et al., 2013).

\section{MEKONG DELTA MODEL}

In 2011 the ambitious plan arose to build a more versatile model for the Mekong Delta. The focus at that time was mainly to get familiar in building a groundwater flow model similar to other large scale modeling studies recently carried out by Deltares. Most of the time, however, was occupied by collecting the necessary data (private and public accessible sources) and transfer them into the iMOD format. It yielded a preliminary 
model for the entire Mekong Delta. This section describes the concepts of the Mekong Delta model at that stage and the improvements made recently. This short description illustrates how easy and transparent it can be to improve a model made in iMOD whenever renewed data becomes available. Any legend has been left out on purpose, since the figures have an illustrative meaning only.

DEM: In 2011 a surface elevation model (DEM) on a resolution of 1,000 meter was downloaded from the internet (http:// www1.gsi.go.jp/ geowww/globalmapgsi/gtopo-30/gtopo30.html), see figure 3-top. The model needed to be able to compute at least the lowlying area in the Mekong Delta solely. We defined the area of interest as those areas where the DEM was less than $15 \mathrm{~m}+$ MSL assuming that the bedrock was outcropping above this elevation. Currently, we achieved a geological map for the Mekong Delta (Anderson, 1978) and we were able to define the model boundaries better. Moreover, we downloaded a DEM on a resolution of 90 meter worldwide (ftp://e0srp01u. ecs.nasa.gov/srtm/version2/SRTM3). This data file has been used to update the top of the hydrological system, as well as the drainage levels throughout the Mekong Delta, see figure 3-bottom.

Boreholes: The subsoil of the groundwater flow model was built from boreholes provided by DWRPIS (Division For Water Resources Planning and Investigation for the South of Vietnam) (DGMS, 2004), see figure 4-left. In totally 95 boreholes were available for which each borehole was categorized by aquifer number. With this data input, we could use the iMOD SolidTool to create a solid of the subsoil by connecting
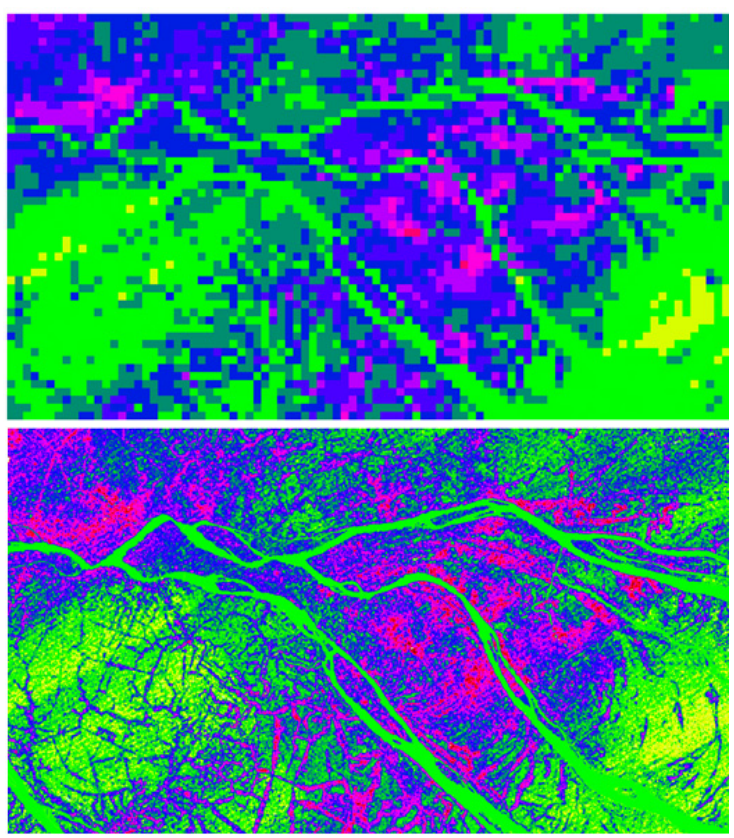

Figure 3. (top) DEM at a scale of 1,000 meter corresponding interfaces between the boreholes, see and (bottom) the DEM at a scale of 90 meter. figure 4-right. Moreover, scanned geological sections were included (figure 4-middle) to incorporate more detail; such as out interfingering of aquitards as well as local modification of interfaces between boreholes. For the Ho Chi Minh City area we achieved several extra boreholes to improve the solid of the subsoil locally.

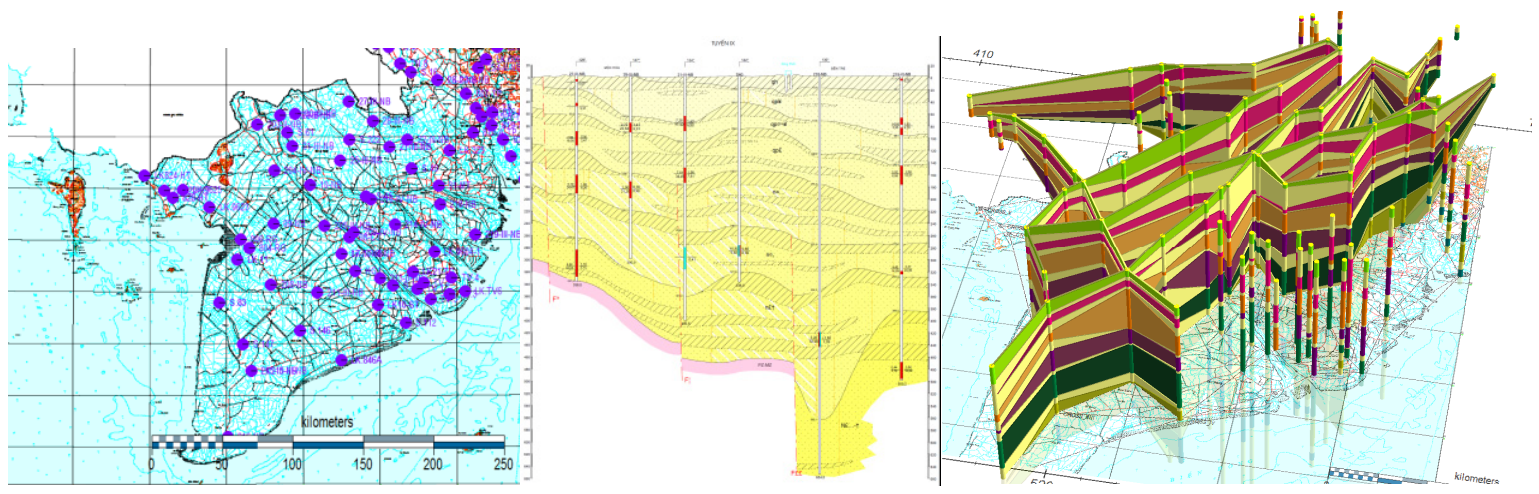

Figure 4. (left) The location of boreholes; (middle) an example of the geological interpretation used in the solid building; (right) the solid of the subsoil for the Mekong Delta.

Permeability: In 2011 we were using a single assumption for the permeability for each model layer. Recently, more detailed information on the distribution of layer permeabilities became available which were used to update the permeability field for several aquifers/aquitards.

Extractions: Extraction rates for industry and domestic usage were estimated roughly in 2011 and updated by a better estimation in 2013. In 2011 we were using 3095 wells, taking 55 million $\mathrm{m}^{3} /$ year as in 2013 we included another 664 wells in Can Tho City and 884 wells in Ho Chi Minh City, taking a total of 33.7 million and 67.3 million $\mathrm{m}^{3} /$ year, respectively. 
Surface water and Drainage systems: Lacking any detailed information about the large river systems in 2011, we generalized those in the Mekong Delta (Mekong and Saigon River) by a simple line representation. The renewed DEM however, shows a very accurate spatial distribution of those rivers and gives a fairly good estimate of its level, see figure 3bottom. In 2013 we developed a SOBEK model for Ho Chi Minh City and surroundings including the Saigon River (Deltares, 2013) that was used to improve the interaction between the river system and the groundwater system. For
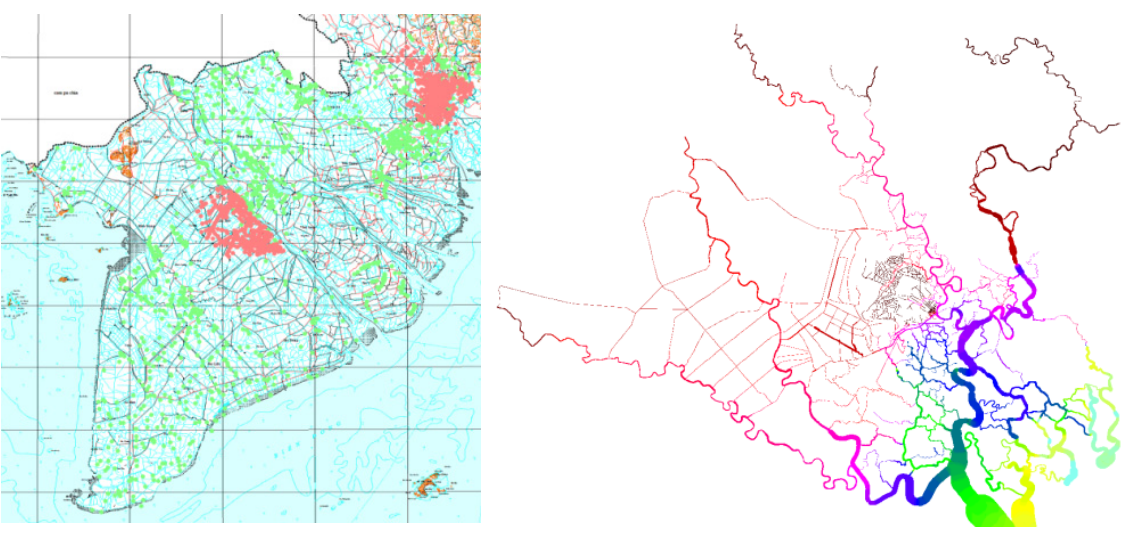

Figure 5. (left) Extractions in 2011 (green) and added in 2013 (red); (right) the outline of the surface water elements Ho Chi Minh City. the open drainage sys-

tems (small canals) in all other areas we assumed that those areas are drained with an offset of 0.25 metersurface level. From field observations (main rice agriculture) this is a fairly appropriate assumption.

\section{RESULTS}

Regional Mekong Delta Model: The entire Mekong Delta model has been simulated on a coarse scale of 1,000 meter, by a simulation network of 308 columns and 410 rows and 8 model layers. It should be noticed that there was no need to scale all input data beforehand to this 1,000 meter scale since iMOD scales internally during the simulation. In the configuration file for iMOD (i.e. a runfile) the scale size is specified as well as the simulation window. In this way it is easy to configure quickly a large variety of models for different scales and simulation windows, interactively. In figure 6-left the results for this 1,000 meter model are presented. In figure 6-middle the layout of the 33 sub-models is depicted that used to create a final model result for the entire Mekong Delta at a scale of 100 meter. The extent of each sub-model overlaps the adjacent submodels by 15,000 meter. This degree of overlap yielded an optimal trade-off between accuracy and efficiency.
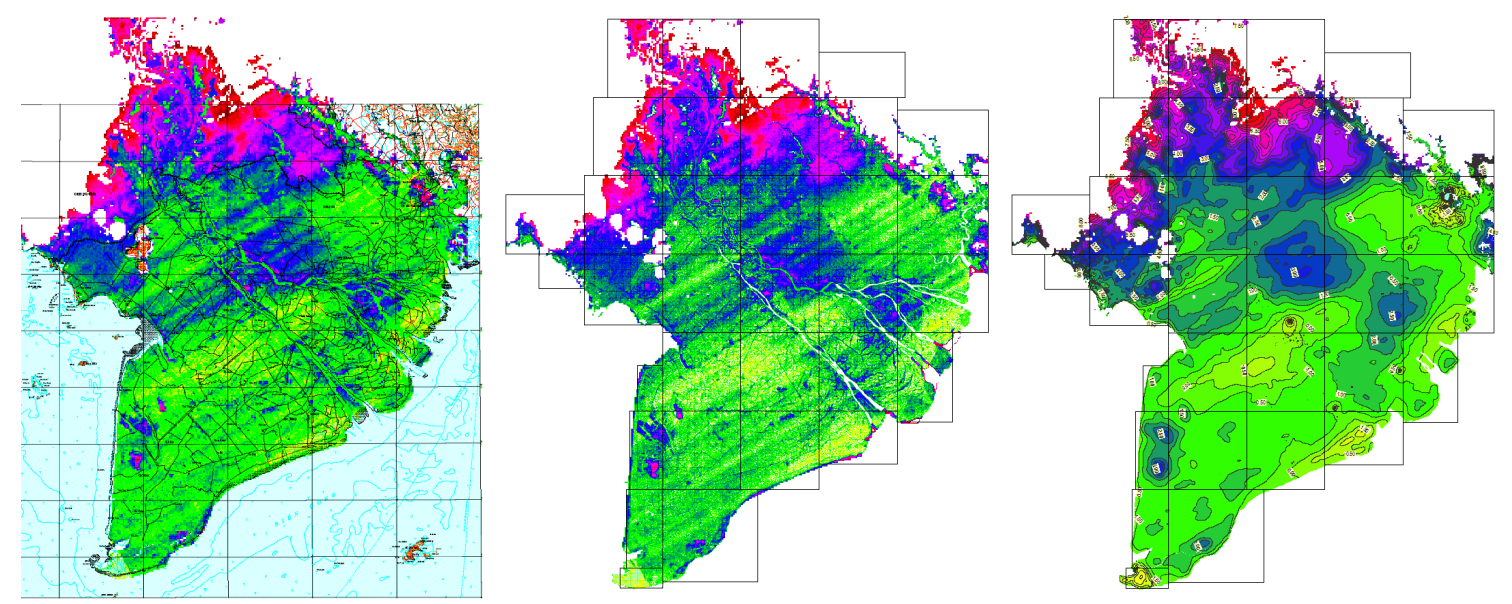

Figuur 6. (left) Simulated phreatic heads for the entire Mekong Delta (1,000 meter) and (middle/right) the 33 sub models used to compute at a scale of a 100 meter.

Each steady-state sub-model ( $600 \times 600 \times 8$ rows, columns and layers on average) took approximately 1.5 minute to complete and was run in parallel with the other sub models. Afterwards all results (heads and budget terms) were merged by iMOD to construct images of the entire computed hydraulic heads for the Mekong Delta on a scale of 100 meter as shown in Figure 6-middle. Figure 6-right shows the hydraulic head for model layer 4 which contains most of the extraction wells. The total number of rows and columns in this result grid became eventually 3,080 and 3,660. Taken the 8 model layers into account we were able to simulate this groundwater flow model within several minutes for 90 million computational nodes. This would take 
992 million floating reals to compute that would take 3.6 GigaBytes of RAM, which is hardly available on a regular PC and requires a 64-bits computer. Moreover, such a huge single model would take 12 hours to solve which can be very dreadful whenever many scenarios need to be computed. With the iMOD approach of using sub models, these large models can be simulated on regular 32 bits computer; fast and easy.

Local Can Tho City Submodel: The process outlined in the previous section has been repeated to invest the drawdown caused by the extraction wells in the Mekong Delta as depicted in figure 5left. So, first the entire regional model was simulated on a coarse scale $(1,000$ meter) with and without those extraction wells to get a global image of the drawdown caused by the extractions(figure 7left). Thereafter both results were used as boundary conditions for a single sub model (50 meter scale with $1,400 \times 1,600$ rows
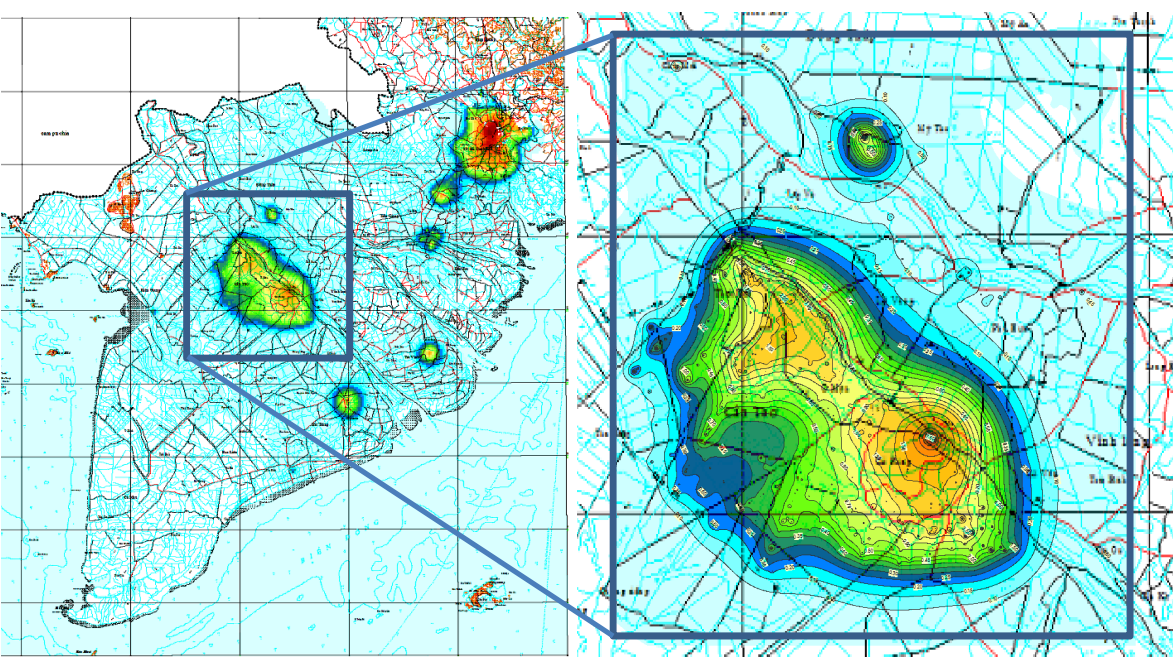

Figure 7. (left) Computed regional drawdown at a scale of 1,000 meter and (right) the computed drawdown at the Can Tho City region at a finer scale of 50 meter for a sub model. Both drawdowns are displayed for model layer 4. and columns) around

Can Tho City to simulate the same scenarios as carried out by the coarse simulation. Based upon these finer simulations we were able to identify the drawdown near specific wells in more detail. The difference between the coarse scale drawdown and the fine scale drawdown could be several meters.

Local Ho Chi Minh City Submodel: For Ho Chi Minh City we have included a detailed surface water model (Deltares, 2013) to simulate the interaction between surface water and groundwater. After we simulated the regional model for the Mekong Delta we included this data type (see figure 5-right) in the runfile for iMOD and simulated a model on a scale of 50 meter. Figure 8-left shows the actual area of interest which is located at the utmost boundary of the Mekong Delta model. Figure 8-middle shows an accurate image of the simulated hydraulic heads which are clearly penetrated by the Mekong River. Figure 8-right shows the declination of the hydraulic head in model layer 4 in which the capture zone of the extraction wells can be discovered. Due to the heavy clay on top of the hydrologic system, deeper, confined aquifers are very sensitive to overexploration, causing drawdowns that reach steady-state conditions after long periods.

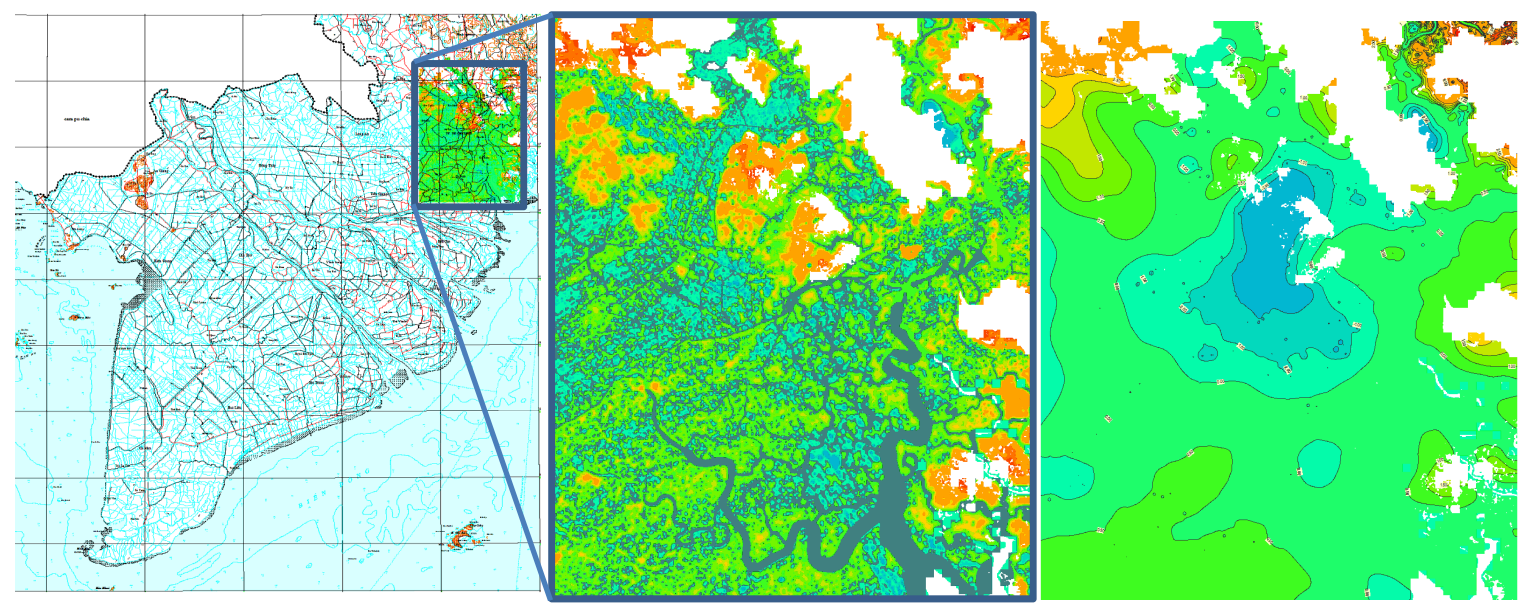

Figure 8 (left) the location of the sub model (blue rectangle) within the regional model for the Mekong Delta that was used to simulate the local hydraulic heads for Ho Chi Minh City as shown in the middle, to the right it shows an accurate representation of the hydraulic head for model layer 4.

Both examples demonstrate the key concepts of modeling with iMOD very well. 


\section{DISCUSSION AND CONCLUSION}

In the near future the model will be further improved in order to be used in normal operational practice. Model evaluation (including model calibration, validation and uncertainty assessment) is one of the first jobs that must be executed. To update the model furthermore, a central model station will be located at one of the participating institution. DWRPIS is the best option since the Division regular receives groundwater investigation assignments from provincial governments in the Mekong Delta region. In addition, research institutions like Deltares, VNU-IER or Can Tho University should also provide technical support to DWRPIS. Improving the geology database of the model could be another direction to improve the model, since, with the hydro-geological map at 1:500.000 scale as a basis, we hardly find any top aquifer, most of the recharge is drained by the drains and hardly any recharge is available to the deeper aquifers. When the model is ready to be used, stakeholders like DWRM of Ho Chi Minh City and provincial agencies in the Mekong delta can acquire specific model applications for their current and future practical challenges such as sustainable groundwater extraction, salt intrusion, and land subsidence.

\section{ACKNOWLEDGMENTS}

DWRPIS for data provided through the geological mapping project coordinated by DGMS (2004). The Vietnam Facility project "Advanced training in modeling and information management applications for water, environmental management and climate change adaptation issues" of Deltares and partners.

\section{REFERENCES}

Anderson, H. R. (1978). "Hydrogeologic reconnaissance of the Mekong Delta in South Vietnam and Cambodia." USGS Water Supply Paper: 1608-R.

Anderson, M. P. and W. W. Woessner (1992). Applied Groundwater Modeling: Simulation of Flow and Advective Transport Inc. San Diego, CA, Academic Press.

Berendrecht, W. L., J. J. J. C. Snepvangers, et al. (2007). Methodology for Interactive Planning for Water Management. MODSIM 2007 International Congress on Modelling and Simulation. Modelling and Simulation Society of Australia and New Zealand. L. Oxley and D. Kulasiri: 74-80. ISBN: 978-9709758400-9758404-9758407.

Boehmer, W. (2000). Ground Water Study Mekong Delta. Modelling report., HASKONING B. V. Consulting Engineers and Architects, in association with Division of Hydro-Geology and Engineering Geology for the South of Vietnam and ARCADIS Euroconsult: 136.

Danh, V. T. (2008). Household Switching Behavior In The Use Of Groundwater in The Mekong Delta, Vietnam, Cantho University, School of Economics and Business Administration. Cantho city, Vietnam: Economy and Environment Program for Southeast Asia (EEPSEA).

Deltares, 2013; Internal Reports Ho Chi Minh City Flood and Inundation Management project

DGMS (2004). Research of geological structure and classification of N-Q sediments in Mekong Delta, Edited by Nguyen, H.D. , DGMS [Division for Geological Mapping for the South of Vietnam].

Eugene, D. M. (1971). "Use of Ground Water in Developing the Mekong Delta, Republic of Viet Nam." Groundwater 9(1).

IPPC (2012). Managing the Risks of Extreme Events and Disasters to Advance Climate Change Adaptation. Cambridge, Cambridge University Press.

IUCN (2011). Groundwater in the Mekong Delta. Discussion paper., IUCN - International Union for the Conservation of Nature: 11.

Minnema, B., P. T. M. Vermeulen, et al. (2013). Utilization of Interactive MODeling (iMOD) to Facilitate Stakeholder Engagement in Model Development Using a Sustainable Approach with Fast, Flexible and Consistent Sub-Domain Modeling Techniques. MODFLOW AND MORE 2013: TRANSLATING SCIENCE INTO PRACTICE. Colorado, The United State of America.

Syvitski, J. P. M., A. J. Kettner, et al. (2009). "Sinking deltas due to human activities - Progress article." Nature Geoscience: 681-686.

Vermeulen, P. T. M. (2006). Model-Reduced Inverse Modeling. Ph.D. thesis, Delft University of Technology - with ref. - with summary in Dutch. ISBN-10: 90-9020536-5. ISBN-13: 978-90-9020536-6.

Vermeulen, P. T. M. (2013). iMOD version 2.7 - Interactive MODeling, Manual, Deltares.

Wagner, F., T. V. Bui, et al. (2012). Chapter 7: Groundwater Resources in the Mekong Delta: Availability, Utilization and Risks. The Mekong Delta System: Interdisciplinary Analyses of a River Delta. F. G. Renaud and C. Kuenzer, Springer: $201-220$. 